\title{
PAPER
}

\section{Neuropsychiatric symptoms in patients with Parkinson's disease and dementia: frequency, profile and associated care giver stress}

\author{
D Aarsland, K Brønnick, U Ehrt, P P De Deyn, S Tekin, M Emre, J L Cummings
}

See Editorial Commentary, p 2

J Neurol Neurosurg Psychiatry 2007;78:36-42. doi: 10.1136/jnnp.2005.083113

See end of article for authors' affiliations

.....................

Correspondence to: D Aarsland, Centre for Clinical Neuroscience Research, Stavanger University Hospital, Stavanger, Norway; daa@sir.no

Received 28 October 2005 Revised version received 6 May 2006 Accepted 9 May 2006 Published Online First 4 July 2006

Objective: To explore the profile of neuropsychiatric symptoms in patients with dementia associated with Parkinson's disease (PDD).

Methods: 537 patients with PDD drawn from an international multicentre clinical trial of rivastigmine were assessed using the 10-item Neuropsychiatric Inventory (NPI). A cluster analysis was used to investigate the inter-relationship of NPI items. Associations between the clusters and demographic and clinical variables were analysed.

Results: $89 \%$ of the patients presented at least one symptom on the NPI, 77\% had two or more symptoms and $64 \%$ had at least one symptom with a score $\geqslant 4$. The most common symptoms were depression (58\%), apathy $(54 \%)$, anxiety $(49 \%)$ and hallucinations (44\%). Patients with more severe dementia and advanced Parkinson's disease had more neuropsychiatric symptoms. Nearly $60 \%$ of the care givers reported at least one NPI symptom to be of at least moderate severe distress. Five NPI clusters were identified: one group with few and mild symptoms (52\%); a mood cluster (11\%, high scores on depression, anxiety and apathy); apathy (24\%; high apathy and low scores on other items); agitation (5\%, high score on agitation and high total NPI score); and a psychosis cluster (8\%; high scores on delusions and hallucinations). The psychosis and agitation clusters had the lowest Mini-Mental State Examination score and the highest Unified Parkinson's Disease Rating Scale and care giver distress scores.

Conclusion: Neuropsychiatric symptoms are common in patients with PDD. The profile of these symptoms differs from that in other types of dementia. Subgroups with different neuropsychiatric profiles were identified. These subgroups may be associated with distinct neurobiological changes, which should be explored in future studies.

A wide range of neuropsychiatric disturbances commonly


Neuropsychiatric disturbances contribute considerably to reduced quality of life, ${ }^{3}$ distress for the care giver ${ }^{4}$ and increased risk for admission to nursing home ${ }^{56}$ in patients with Parkinson's disease. Most patients with Parkinson's disease will eventually develop dementia, ${ }^{7}$ and neuropsychiatric symptoms are more common in those with Parkinson's disease with dementia (PDD). ${ }^{18}$ Knowledge of the wide variety of psychiatric symptoms and diagnostic skills to identify and implement optimal treatment of these symptoms are thus of major importance in the management of patients with Parkinson's disease and those with PDD.

The multiple psychiatric symptoms in patients with dementia tend to cluster into discrete psychiatric syndromes, ${ }^{9}{ }^{10}$ indicating that the underlying pathophysiological constructs may explain the relationship between observed variables. Identifying these underlying constructs is important, as it may prove to be more valuable to correlate neurochemical measures with syndromes rather than with individual symptoms. Also, from the clinical point of view, treatment might be best directed towards syndromes rather than towards each specific, individual behavioural symptom. Finally, there is emerging evidence of subtypes within the major neurodegenerative disorders, including Parkinson's disease, ${ }^{11}$ and exploring psychiatric syndromes may help in distinguishing such subtypes.

Statistical methods such as factor analysis, cluster analysis and latent class analysis have been used to identify empirically based classifications of neuropsychiatric clusters in patients with neurodegenerative disorders, with high face validity. ${ }^{10}{ }^{12}$ These empirically based classifications could enhance our understanding of the heterogeneity of patients with dementia, and lead to clearer treatment strategies for different subgroups. Although factor analysis can provide information on how symptoms correlate in a sample, it is of limited value in understanding how the symptoms occur in groups of patients, and is thus of limited value in identifying groups of patients on the basis of symptoms. Rather, classification of individual patients is possible using cluster analysis, a data-driven, exploratory classification method. ${ }^{13}$

Five different clusters of neuropsychiatric symptoms were recently identified in patients with Parkinson's disease. ${ }^{12}$ However, to the best of our knowledge, no studies have explored how neuropsychiatric symptoms cluster in patients with PDD. Therefore, to explore whether natural subgroups of patients with Parkinson's disease can be identified on the basis of their neuropsychiatric profile, we administered the Neuropsychiatric Inventory (NPI) to a large sample of patients with PDD, and used cluster analysis to identify the interrelationship of neuropsychiatric symptoms. The analysis is based on the baseline data obtained in a large phase III clinical trial evaluating the safety and efficacy of rivastigmine in PDD. ${ }^{14}$

\section{METHODS}

\section{Patients}

Men and women at least 50 years old, with a clinical diagnosis of Parkinson's disease according to the UK Parkinson's Disease

Abbreviations: MMSE, Mini-Mental State Examination; NPI,

Neuropsychiatric Inventory; PDD, dementia associated with Parkinson's disease; UPDRS, Unified Parkinson's Disease Rating Scale 
Society Brain Bank clinical diagnostic criteria, ${ }^{15}$ and of PDD according to the fourth edition of the Diagnostic and statistical manual of mental disorders (Code 294.1), ${ }^{16}$ were recruited from research centres in Austria, Belgium, Canada, France, Germany, Italy, The Netherlands, Norway, Portugal, Spain, Turkey and the UK. ${ }^{14}$ Patients were required to have mild to moderately severe dementia as defined by a Mini-Mental State Examination $(\mathrm{MMSE})^{17}$ score of 10-24 inclusive, with the onset of dementia at least 2 years after the first diagnosis of idiopathic Parkinson's disease. Patients were required to have contact with a responsible care giver on at least 3 days a week. For ambulatory patients receiving professional care (eg, living in a nursing home), the care giver could be a designated member of the medical team. Exclusion criteria included any primary neurodegenerative disorder other than Parkinson's disease or any other causes of dementia; a major depressive episode; active, uncontrolled seizure disorder; any disability or unstable disease that might prevent the patient from completing all study requirements; and a known hypersensitivity to drugs similar to rivastigmine in structure or pharmacological action.

The study was approved by local properly constituted institutional review boards. Participants and their care givers gave written informed consent. All procedures were in accordance with ethical standards of the responsible committee on human experimentation and with the Helsinki declaration.

\section{Clinical evaluation}

Neuropsychiatric symptoms were assessed using the 10-item $\mathrm{NPI}^{18}$ by a trained rater following standard procedures. The validity of the NPI has been established, ${ }^{18}$ and high reliability in Parkinson's disease has been reported. ${ }^{1}$ Firstly, screening questions for each of the 10 neuropsychiatric symptoms were asked. Positive responses were probed with structured questions focusing on specific features of the neuropsychiatric symptom. The informant rated the frequency of each symptom on a scale from 1 to 4 , and the severity of the symptom on a scale from 1 to 3. A composite score, defined as the product of frequency and severity, yielding a score ranging from 1 to 12 for each item, was used in the analysis. A care giver distress item is also included, where the care giver is requested to score the level of emotional distress experienced by the care giver caused by each symptom (level of distress: not at all, 1; minimal, 2; moderate, 3; severe, 4; very severe or extreme distress, 5).

Cognition was assessed by means of MMSE scores ${ }^{17}$; motor symptoms were evaluated with the Unified Parkinson's Disease
Rating Scale (UPDRS) and motor subscale (part III) ${ }^{19}$; and staging of Parkinson's disease by the Hoehn and Yahr scale. ${ }^{20}$

\section{Statistical analyses}

Descriptive statistics were applied first (mean, standard deviation (SD), rates). Student's t test was used for comparisons of normally distributed continuous data and $\chi^{2}$ test for categorical variables. Non-parametric tests were used for comparison of the NPI scores owing to the skewness and non-linearity of these data.

NPI subscores of each patient with a positive score on at least one NPI item were standardised to z score values based on the mean and SD of each NPI variable, to ensure equal weighting of the different symptoms in the clustering procedure. A similarity matrix was calculated using an euclidian distance measure. Kmeans cluster analysis was used on the similarity matrix. For validation purposes, we used a split-sample validation procedure. The sample was divided into two, using the randomisation procedure in SPSS V.12.01. Separate similarity matrices were computed for each sample and subjected to a K-means clustering procedure. The resulting clusters were compared with the clusters from the full-sample analysis regarding classification agreement.

The clusters were analysed regarding the Hoehn and Yahr stage, MMSE and NPI care giver distress scale scores. One-way analysis of variance was used for statistical comparisons of the five clusters for continuous variables, with retrospective corrections where appropriate, using Scheffe's test. A value of $\mathrm{p}<0.05$ was considered significant. In addition, a multinomial logistic regression analysis was carried out to study the effect of clinical features on the allocation of a patient to a particular cluster, with cluster number as the dependent variable and clinical and demographic variables as predictors.

\section{RESULTS}

\section{Sample characteristics}

NPI was completed for 537 patients (65\% men and 35\% women). The mean (SD) age of the sample was 72.64 (6.61) years, and they had 9.00 (4.06) years of formal education. The mean (SD) duration since onset of Parkinson's disease was 10.03 (5.9) years, and that since diagnosis of dementia was 2.15 (1.7) years. The mean MMSE score was 19.33 (3.90), UPDRS motor score 33.32 (14.07) and Hoehn and Yahr stage 2.78 (0.83). All patients received antiparkinsonian agents, $26.6 \%$ used an antidepressant, 27.4\% used an antipsychotic

Table 1 Neuropsychiatric Inventory item and care giver distress scores in the total group and in those showing symptoms

\begin{tabular}{|c|c|c|c|c|c|c|c|c|c|c|c|c|c|}
\hline \multirow[b]{3}{*}{$\begin{array}{l}\text { Neuropsychiatric } \\
\text { Inventory } \\
\text { item }\end{array}$} & \multicolumn{4}{|c|}{ All patients } & \multicolumn{9}{|c|}{ Patients showing symptoms } \\
\hline & \multicolumn{2}{|l|}{$\begin{array}{l}\text { Item } \\
\text { score }\end{array}$} & \multicolumn{2}{|l|}{$\begin{array}{l}\text { Care giver } \\
\text { distress } \\
\text { score }\end{array}$} & \multirow{2}{*}{$\begin{array}{l}\begin{array}{l}\text { Proportion } \\
\text { with non-zero } \\
\text { score }\end{array} \\
\text { n (\%) }\end{array}$} & \multicolumn{2}{|l|}{$\begin{array}{l}\text { Proportion } \\
\text { with } \\
\text { score } \geqslant 4\end{array}$} & \multicolumn{2}{|l|}{$\begin{array}{l}\text { Item } \\
\text { score }\end{array}$} & \multicolumn{2}{|c|}{$\begin{array}{l}\text { Care giver } \\
\text { distress } \\
\text { score }\end{array}$} & \multirow[b]{2}{*}{$\begin{array}{l}\text { Number } \\
(\%)^{*} \text { with } \\
\text { score } \geqslant 3\end{array}$} & \multirow[b]{2}{*}{$\begin{array}{l}\text { Percentage } \\
\text { of all }\end{array}$} \\
\hline & Mean & SD & Mean & SD & & n $(\%)^{*}$ & $\begin{array}{l}\text { Percentage } \\
\text { of all }\end{array}$ & Mean & SD & Mean & SD & & \\
\hline Delusions & 1.03 & 2.29 & 0.64 & 1.3 & $132(24.6)$ & $69(52)$ & 12.8 & 4.21 & 2.9 & 2.58 & 1.4 & $80(61)$ & 14.9 \\
\hline Hallucinations & 1.48 & 2.34 & 0.89 & 1.4 & $236(43.9)$ & 87 (37) & 16.2 & 3.35 & 2.3 & 2.03 & 1.4 & $93(39)$ & 17.3 \\
\hline $\begin{array}{l}\text { Agitation/ } \\
\text { aggression }\end{array}$ & 1.05 & 2.07 & 0.75 & 1.3 & $175(32.6)$ & $58(33)$ & 10.7 & 3.20 & 2.5 & 2.29 & 1.5 & $79(45)$ & 14.7 \\
\hline Depression & 1.96 & 2.57 & 1.31 & 1.5 & $309(57.5)$ & $116(38)$ & 21.4 & 3.40 & 2.6 & 2.27 & 1.3 & $142(46)$ & 26.2 \\
\hline Anxiety & 1.93 & 2.72 & 1.07 & 1.4 & $263(49.0)$ & $120(46)$ & 22.2 & 3.95 & 2.7 & 2.19 & 1.2 & $114(43)$ & 21.1 \\
\hline Euphoria & 0.10 & 0.67 & 0.05 & 0.3 & $20(3.7)$ & $6(30)$ & 1.1 & 2.80 & 2.2 & 1.45 & 1.1 & $4(20)$ & 0.7 \\
\hline Apathy & 2.95 & 3.43 & 1.25 & 1.5 & $291(54.3)$ & $203(70)$ & 37.7 & 5.40 & 2.9 & 2.30 & 1.3 & $145(50)$ & 27.0 \\
\hline Disinhibition & 0.34 & 1.20 & 0.23 & 0.8 & $63(11.7)$ & $20(32)$ & 3.7 & 2.90 & 2.2 & 1.95 & 1.5 & $25(40)$ & 4.6 \\
\hline $\begin{array}{l}\text { Irritability/ } \\
\text { lability }\end{array}$ & 1.04 & 2.21 & 0.65 & 1.2 & $159(29.7)$ & $60(38)$ & 11.2 & 3.51 & 2.8 & 2.22 & 1.4 & $69(43)$ & 12.8 \\
\hline $\begin{array}{l}\text { Aberrant motor } \\
\text { behaviour }\end{array}$ & 1.05 & 2.40 & 0.46 & 1.1 & $118(22.0)$ & $68(58)$ & 12.6 & 4.77 & 2.9 & 2.11 & 1.4 & $52(44)$ & 9.7 \\
\hline Total NPI & 12.93 & 12.04 & 7.30 & 6.7 & $480(89.4)$ & $345(72)$ & 64.1 & 14.27 & 11.9 & 8.05 & 8.7 & $315(66)$ & 58.6 \\
\hline
\end{tabular}

*Percentage of patients showing symptoms. 


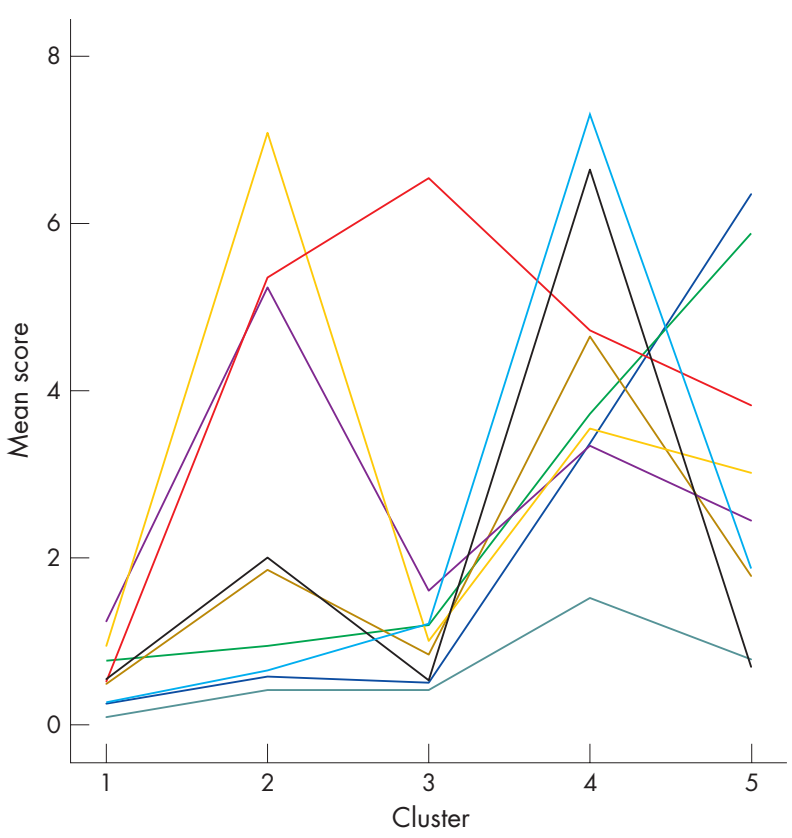

\begin{tabular}{|lll|}
\hline - Delusions & - Depression/dysphoria & - Disinhibition \\
- Hallucinations & - Anxiety & - Irritability/lability \\
- Agitation/aggression & - Apathy/indifference & - Aberrant motor behaviour \\
\hline
\end{tabular}

Figure 1 The final cluster solution: mean Neuropsychiatric Inventory item scores in the five clusters.

agent and $19.5 \%$ used an anxiolytic agent; $13.1 \%$ used more than one type of psychotropic drug.

\section{NPI scores}

Table 1 shows the mean score and proportion of patients with a non-zero score on the NPI items. The mean (SD) total NPI score was 12.9 (12.0). The most common symptoms were depression, apathy, anxiety and hallucinations, and the least common were euphoria and disinhibition. Only $10.6 \%$ were without any symptoms, whereas $89.4 \%$ presented at least one symptom. Seventy three per cent of those who presented with at least one symptom, or $64 \%$ of the total group, had at least one symptom with a score $\geqslant 4$. The mean number of symptoms present was 3.3 (2.2), and $60 \%$ displayed $\geqslant 3$ symptoms. Among those patients who had symptoms, the highest scores were found for apathy, delusions, anxiety and depression.

\section{Associations between NPI and clinical characteristics}

The total NPI score did not differ between male and female patients $(p=0.55)$, and we found no association with age $(\mathrm{r}=-0.08 ; \mathrm{p}=0.06)$. However, we found significant associations between total NPI score and cognitive impairment and stage of Parkinson's disease. Patients with an MMSE score below the median score of 20 had more neuropsychiatric symptoms than those with MMSE score $>20$ : mean total NPI 15.02 (13.23) v 11.19 (10.71); number of symptoms 3.7 (2.3) $v$ 3.0 (2.0); Mann-Whitney test; $\mathrm{p}=0.001$ for both). Comparing individual items, patients with MMSE score $<20$ had higher mean scores and a higher proportion of non-zero scores for the variables delusions, hallucinations, aberrant motor behaviour $(\mathrm{p}<0.001$ for all three) and apathy $(\mathrm{p}=0.006)$.

A similar pattern was found for the relationship between NPI items and Hoehn and Yahr stage. Those with a Hoehn and Yahr stage at or above the median stage of 3 had a total NPI score of 15.06 (13.14) compared with $10.79(10.50)$ in those with a Hoehn and Yahr score $<3 \quad(\mathrm{p}<0.001)$. Patients with more advanced Parkinson's disease had higher scores and a higher proportion of non-zero scores on the items delusions $(\mathrm{p}<0.001)$, hallucinations $(\mathrm{p}<0.001)$, apathy $(\mathrm{p}=0.001)$ and aberrant motor behaviour $(\mathrm{p}=0.008)$.

\section{NPI cluster analysis}

The cluster analysis classified the patients in five clusters on the basis of a judgement of the interpretability of the solution (fig 1). The split-sample validation procedure gave a total classification agreement of $85 \%$. The agreement between the cluster classification in the first and second half of the sample versus the full-sample solution was $91 \%$ and $79 \%$, respectively.

The patients in cluster 1 , the largest group $(n=279 ; 52.1 \%)$, consisted principally of participants reporting few and mild neuropsychiatric symptoms. The mean (SD) total NPI score of this group was $5.2(4.6)$, and $64.3 \%$ showed $<3$ symptoms. Cluster $2(\mathrm{n}=58 ; 10.8 \%)$ was characterised by patients with high scores on depression, anxiety and apathy, and low scores on the other items. Owing to their symptom profile, we refer to this as the "mood" group. Cluster $3(\mathrm{n}=126 ; 23.5 \%)$, had high scores on apathy (mean $6.6(2.1)$ ) and low scores on the remaining items, including depression, thus labelled the "apathy" group. A small group $(\mathrm{n}=29 ; 5.4 \%)$, cluster 4 , consisted of patients with moderate or severe subscores on most items, including irritability and agitation items. Scores on irritability and agitation were low in the other clusters. This group was labelled the "agitation" group, with a total NPI score of 39.2 , and with $55.2 \%$ showing $\geqslant 6$ symptoms. The final cluster, cluster $5(\mathrm{n}=45 ; 8.4 \%)$, was characterised by high scores on visual hallucinations $(5.9(2.9))$ and delusions (6.4 (2.7)), in combination with lower scores on most other items, and was labelled the "psychosis" group. The differences in total NPI score between the groups were significant $(\mathrm{F}=285$, $\mathrm{df}=4532, \mathrm{p}<0.00 \mathrm{l}$; post-hoc Tukey test, all $\mathrm{p}$ values $<0.001$ ), with the exception of clusters 2 and 5, not significant (fig 2).

We found slight differences between the clusters with regard to sex distribution $(\mathrm{p}<0.05)$ : the proportion of men was lowest in the mood group (50.9\%) and highest in the apathy group

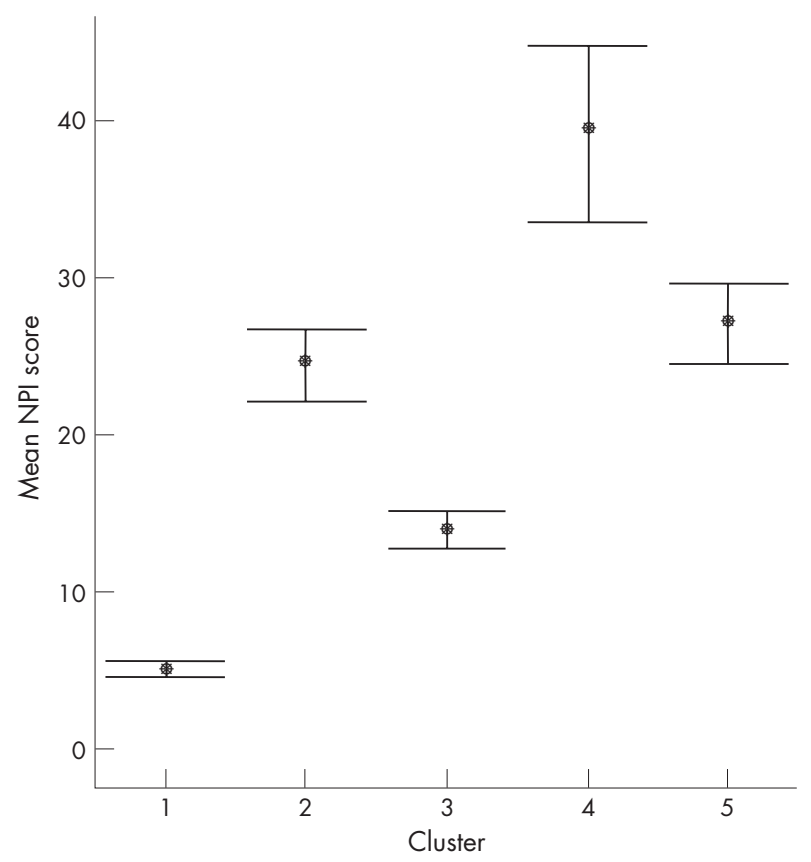

Figure 2 Total Neuropsychiatric Inventory (NPI) scores in the five neuropsychiatric clusters. $\bullet$, mean score. Error bars show $95 \%$ confidence interval of mean. 


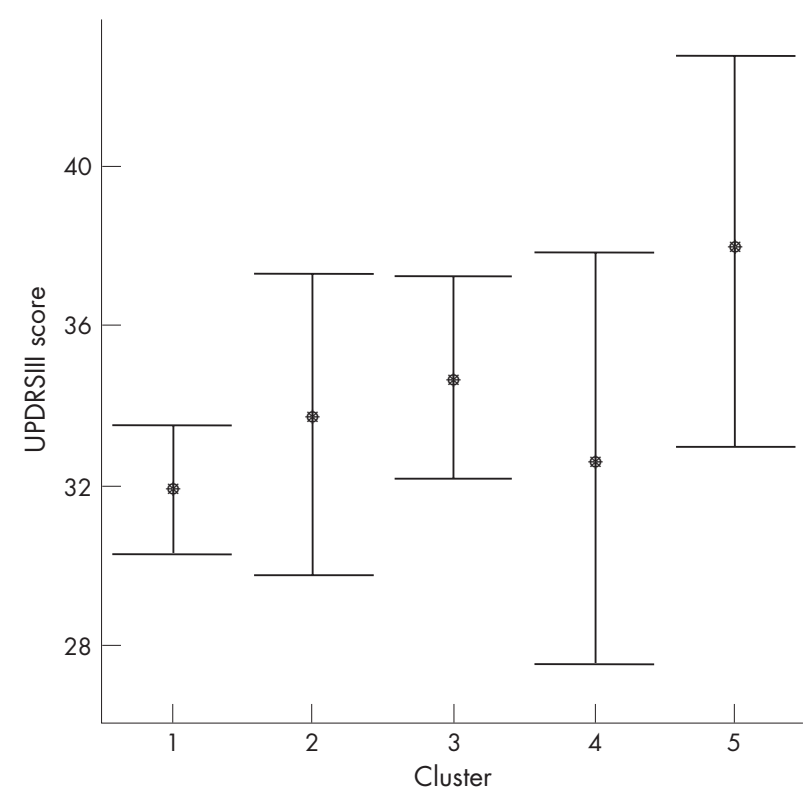

Figure 3 Mean Unified Parkinson's Disease Rating Scale-third edition (UPDRS III) motor subscores in the five neuropsychiatric clusters. $\bullet$, mean score. Error bars show $95 \%$ confidence interval of mean.

(74.6\%). The set of one-way analyses of variance showed that the five clusters did not differ regarding age $(\mathrm{F}=1.9, \mathrm{df}=4$, $\mathrm{p}=0.12)$ or duration of Parkinson's disease $(\mathrm{F}=1.3, \mathrm{df}=4$, $\mathrm{p}=0.25)$. The psychosis group had the highest mean UPDRS motor subscore (fig 3), but there were no significant betweengroup differences $(\mathrm{F}=2.3, \mathrm{df}=4, \mathrm{p}=0.06)$. We found, however, highly significant differences among the clusters regarding MMSE $(\mathrm{F}=6.1, \mathrm{df}=4, \mathrm{p}<0.001$; fig 4): cluster 1 , with minimal neuropsychiatric disturbances, and the Mood and Apathy clusters had the highest MMSE scores, and had significantly higher scores than the patients in cluster 4, the Agitation cluster. The MMSE score in the Psychosis cluster was lower than in clusters $1-3$, but these differences did not reach significance, although the difference between clusters 5 and 2 approached significance $(\mathrm{p}=0.057)$. Table 2 shows the odds ratios for effects of these clinical and demographic features on cluster allocation. A low score on the MMSE predicted allocation to clusters 4 and 5 compared with cluster 1, and



Figure 4 Mean Mini-Mental State Examination (MMSE) scores in the five neuropsychiatric clusters. $\bullet$, mean score. Error bars show $95 \%$ confidence interval of mean.

male sex was associated with a higher likelihood of being placed in cluster 3 and lower likelihood of being in cluster 2 than in cluster 1. More advanced disease stage was associated with a significantly higher likelihood of being placed in all clusters with neuropsychiatric symptoms (ie, clusters 2-5) than in cluster 1. The frequency of using antidepressants did not differ among the clusters, but we found a significant difference in the use of antipsychotics $\left(\chi^{2}=28.7, \mathrm{df}=4, \mathrm{p}<0.001\right)$. The highest proportion using antipsychotic agents was in cluster 5 $(56 \%)$, intermediate in clusters $4(34 \%)$ and $3(33 \%)$, and lowest in clusters $2(21 \%)$ and $1(19 \%)$.

\section{NPI care giver distress scores}

As expected, the mean care giver distress score was highest for those items with the highest frequency. In patients with a positive score for that item, the highest care giver distress score was found for delusions, apathy, agitation, depression and irritability (table 1). Moderately severe distress—that is, a score

\begin{tabular}{|c|c|c|c|c|}
\hline Cluster & Variable & $\mathrm{p}$ Value & Odds ratio & $\begin{array}{l}95 \% \text { confidence } \\
\text { interval }\end{array}$ \\
\hline \multirow[t]{4}{*}{2} & Age & 0.053 & 0.957 & 0.916 to 1.001 \\
\hline & Sex & 0.021 & 1.993 & 1.109 to 3.579 \\
\hline & Hoehn and Yahr stage & 0.019 & 1.563 & 1.076 to 2.271 \\
\hline & MMSE score & 0.072 & 1.082 & 0.993 to 1.178 \\
\hline \multirow[t]{4}{*}{3} & Age & 0.845 & 1.003 & 0.970 to 1.038 \\
\hline & Sex & 0.029 & 0.586 & 0.363 to 0.946 \\
\hline & Hoehn and Yahr stage & .010 & 1.425 & 1.088 to 1.867 \\
\hline & MMSE score & 0.470 & 0.979 & 0.924 to 1.037 \\
\hline \multirow[t]{4}{*}{4} & Age & 0.006 & 0.922 & 0.869 to 0.977 \\
\hline & Sex & 0.696 & 1.175 & 0.523 to 2.636 \\
\hline & Hoehn and Yahr stage & 0.024 & 1.716 & 1.072 to 2.746 \\
\hline & MMSE score & 0.001 & 0.850 & 0.772 to 0.935 \\
\hline \multirow[t]{4}{*}{5} & Age & 0.182 & 0.967 & 0.920 to 1.016 \\
\hline & Sex & 0.797 & 0.915 & 0.467 to 1.795 \\
\hline & Hoehn and Yahr stage & 0.020 & 1.590 & 1.077 to 2.347 \\
\hline & MMSE score & 0.034 & 0.916 & 0.844 to 0.993 \\
\hline
\end{tabular}






Figure 5 Sum of care giver distress scores in the five Neuropsychiatric Inventory (NPI) clusters. •, mean score. Error bars show $95 \%$ confidence interval of mean.

of $\geqslant 3$-was reported by $>20 \%$ of carers for several symptoms, and $58.6 \%$ of the carers reported at least one neuropsychiatric symptom to be causing at least moderately severe distress. Among the five clusters, the highest total care giver distress total score was found for the agitation, psychosis and mood clusters, but even the distress score for the apathy cluster was significantly higher than the distress score for the cluster, showing no or few NPI symptoms (fig 5).

\section{DISCUSSION}

We describe the neuropsychiatric symptoms in the largest sample of patients with PDD reported to date. At least one neuropsychiatric symptom was present in nearly $90 \%$ of the included participants. Although most patients had few symptoms and low scores, a considerable number of patients had many symptoms, and $64 \%$ had a composite score of $\geqslant 4$ for at least one item, indicating at least moderate severity. The most common symptoms were depression, anxiety, apathy and hallucinations. The symptoms tended to occur in a specific pattern, and five symptom clusters were identified, dominated by mood symptoms, apathy, psychosis, a small group with predominant agitation accompanied by several other, marked symptoms, and a group with few and mild symptoms. In addition to being common, the clinical relevance of neuropsychiatric symptoms in patients with PDD is further underlined by the finding that nearly $60 \%$ of the care givers reported at least one NPI symptom to be a cause of moderate or severe distress.

The frequency and severity of psychiatric symptoms in this PDD group are higher than those reported in a communitybased cohort of patients with Alzheimer's disease, ${ }^{21}$ and the mean total NPI score is similar to the score in patients with Alzheimer's disease recruited for clinical trials with a cholinesterase inhibitor. ${ }^{22}$ However, the distribution and clustering of psychiatric symptoms differ markedly between patients with Alzheimer's disease and those with PDD, supporting the findings in a previous smaller study. ${ }^{23}$ Firstly, although the identification of groups dominated by mood symptoms, psychosis and agitation in patients with PDD is similar to those in patients with Alzheimer's disease, ${ }^{1024}$ a substantial group $(24 \%)$ with predominantly apathy but with low scores on mood symptoms was identified in patients with PDD, which has not been reported in those with Alzheimer's disease. Secondly, in the psychosis group, hallucinations were twice as common as delusions in patients with PDD, whereas the reverse is true in Alzheimer's disease. The proportion with delusions is similar in patients with Alzheimer's disease and in those with PDD, whereas hallucinations were present in $44 \%$ of patients with PDD compared with only 13\% in those with Alzheimer's disease. ${ }^{21}$ The cause for this difference may lie in differential pathophysiology, as suggested by recent findings on morphological $^{25}$ and neurochemical ${ }^{26}$ substrates of delusions and hallucinations in dementia with Lewy bodies, a disorder with clinical and neurobiological similarities with PDD. ${ }^{27}$ Thus, the brain changes underlying psychosis in Alzheimer's disease may differ from those in PDD, as has previously been shown for dementia with Lewy bodies and Alzheimer's disease. ${ }^{25}$ These findings of different clustering of neuropsychiatric symptoms in Alzheimer's disease and PDD extend previous clinical and neurobiological observations suggesting that dementia in patients with Parkinson's disease is not mainly due to concomitant Alzheimer's disease.

The identification of neuropsychiatric clusters supports and extends previous findings indicating clinical subgroups in patients with Parkinson's disease. ${ }^{1128}$ Several studies have suggested subgroups according to the profile of cognitive impairment, with some patients showing a pattern compatible with frontostriatal deficits and others more with a temporallimbic deficit. ${ }^{29-31}$ Differential neurochemical deficits underlying different motor subtypes have also been previously reported. ${ }^{28}$ On the basis of the current study, we propose subgroups of patients with PDD based on the neuropsychiatric symptom profile, with potential treatment implications. We hypothesise specific neurobiological changes underlying the different neuropsychiatric clusters. Apathy has been linked to pathology of the anterior cingulum, and to disturbances in the medial frontostriatal circuitry, probably mediated by dopaminergic deficits. ${ }^{32}$ Hallucinations in patients with Lewy body disease are associated with Lewy bodies in the temporal cortex, ${ }^{33}$ and with cholinergic deficits, ${ }^{26}$ and thus cholinergic deficits may be particularly pronounced in these patients. Depression in Parkinson's disease has been associated with serotonergic deficits, but also noradrenergic changes. ${ }^{34}$ Finally, agitation is common in Alzheimer's disease, and neuropathological studies have found that neurofibrillar burden in the left orbitofrontal cortex correlates considerably with agitation scores. ${ }^{35}$ Thus, in patients with PDD with agitation, Alzheimer's disease-like changes may be particularly pronounced, although other pathologies in the orbitofrontal cortex may also contribute. In summary, the neuropsychatric profile of patients with PDD may provide information on the differential use of dopaminergic, serotonergic, noradrenergic and cholinergic drugs. However, given the complex anatomical and neurotransmitter interactions in the brain, linking complex behaviours such as psychiatric symptoms to one specific brain area or one transmitter system is clearly an oversimplification, although major anatomical and chemical contributors can be identified. In addition, the MMSE score differed significantly between clusters, and so it is possible that the neuropsychiatric syndromes to some extent may represent different stages of the dementia. Nevertheless, our findings do provide an empirical basis for testing the neurochemical hypotheses in future clinical trials.

Dopaminergic agents may have behavioural effects, ${ }^{36}$ and may thus contribute to the profile of neuropsychiatric symptoms, in particular to the occurrence of visual hallucinations. However, several studies have shown that antiparkinsonian agents are only weakly associated with visual 
hallucinations, suggesting that disease-related factors are more important determinants of psychiatric symptoms in patients with PDD. ${ }^{37}$ Furthermore, antipsychotic drugs were used by 20 $30 \%$ of patients without or with only mild neuropsychiatric symptoms, indicating that these drugs may have influenced the observed symptom profile, and that psychotic symptoms are even more common than observed in this study. A proportion of patients used antidepressants. Although the use of antidepressants did not differ among clusters, it is possible that the use of such drugs might have influenced the NPI profile and the distribution of patients to the different clusters.

We note some methodological limitations of this study. Firstly, as this was a multicentre clinical trial, the inter-rater reliability of NPI may be lower and thus some important associations might have been lost. However, NPI is a highly structured instrument with proved high reliability, and the raters participated in training sessions before the study.

Secondly, recording of neuropsychiatric symptoms was observer based only, and some neuropsychiatric symptoms with more subjective character may have been missed. In addition, some symptoms, such as obsessive-compulsive symptoms, were not assessed.

Thirdly, this was a cross-sectional study, and a longitudinal approach may more accurately reflect the frequency and clustering of neuropsychiatric disturbances. The diagnosis was clinical without autopsy confirmation, and misdiagnosis may occur. However, established diagnostic criteria for Parkinson's disease and standardised assessments of motor symptoms were used. Fourthly, a control group was not included. However, there is evidence that the frequency and severity of NPI items is low in healthy elderly people ${ }^{18}$ and in those without dementia. ${ }^{38}$ With regard to statistical methods, cluster analysis can be criticised because of the subjective nature of several decisions affecting the final outcome. However, the split-sample validation procedure showed an agreement of $85 \%$ between the cluster classification in the subsamples and the full sample, far higher than the $20 \%$ agreement expected if the classification was random. The patterns of relationships with the other clinical variables provide further support for the validity of our clustering solution, as does the face validity and similarity with previous cluster analyses.

The patients were recruited for a clinical trial with rivastigmine. The patients had fairly low NPI scores, and this is typical for trial populations as very disturbed patients cannot participate in trials. Trial participants also tend to be better educated and have better general health, and this may affect the generalisability to other PDD populations. In addition, patients with major depression were not included. However, major depression is rare in Parkinson's disease. ${ }^{39} 40$ Finally, only patients with a carer who must be in contact with the patient for a minimum of 3 days a week were included. Thus, a selection bias may have been introduced. However, the age, severity of motor symptoms, level of cognitive impairment and prevalence, and distribution and severity of psychiatric symptoms are remarkably similar to those of a small communitybased cohort of patients with PDD. ${ }^{23}$ Thus, this group is representative of the overall population with PDD, supporting the validity of the findings.

\section{ACKNOWLEDGEMENTS}

This study was based on baseline data from a drug trial sponsored by the Novartis Pharmaceuticals Corporation.

\section{Authors' affiliations}

D Aarsland, K Brønnick, U Ehrt, Centre for Clinical Neuroscience

Research, Stavanger University Hospital, Stavanger, Norway
P P De Deyn, Department of Neurology, Middelheim Hospital, Laboratory of Neurochemistry and Behavior, Born-Bunge Foundation, University of Antwerp, Antwerp, Belgium

S Tekin, Novartis Pharmaceuticals Corporation, East Hanover, New Jersey, USA

M Emre, Istanbul Faculty of Medicine, Istanbul, Turkey

J L Cummings, UCLA Alzheimer's Disease Center, Los Angeles, California, USA

Competing interests: None declared.

\section{REFERENCES}

1 Aarsland D, Larsen JP, Lim NG, et al. Range of neuropsychiatric disturbances in patients with Parkinson's disease. J Neurol Neurosurg Psychiatry 1999:67:492-6.

2 Brown RG, MacCarthy B. Psychiatric morbidity in patients with Parkinson's disease. Psychol Med 1990;20:77-87.

3 Karlsen KH, Tandberg E, Arsland D, et al. Health related quality of life in Parkinson's disease: a prospective longitudinal study. J Neurol Neurosurg Psychiatry 2000:69:584-9.

4 Aarsland D, Larsen JP, Karlsen K, et al. Mental symptoms in Parkinson's disease are important contributors to caregiver distress. Int J Geriatr Psychiatry 1999; 14:866-74.

5 Aarsland D, Larsen JP, Tandberg E, et al. Predictors of nursing home placement in Parkinson's disease: a population-based, prospective study. J Am Geriatr Soc 2000;48:938-42.

6 Goetz CG, Stebbins GT. Mortality and hallucinations in nursing home patients with advanced Parkinson's disease. Neurology 1995;45:669-71.

7 Aarsland D. Andersen K, Larsen JP, et al. Prevalence and characteristics of dementia in Parkinson disease: an 8-year prospective study. Arch Neurol 2003;60:387-92

8 Emre M. Dementia associated with Parkinson's disease. Lancet Neurol 2003;2:229-37.

9 Lyketsos CG, Sheppard JM, Steinberg M, et al. Neuropsychiatric disturbance in Alzheimer's disease clusters into three groups: the Cache County study. Int J Geriatr Psychiatry 2001;16:1043-53.

10 Mirakhur A, Craig D, Hart DJ, et al. Behavioural and psychological syndromes in Alzheimer's disease. Int J Geriatr Psychiatry 2004;19:1035-9.

11 Foltynie T, Brayne C, Barker RA. The heterogeneity of idiopathic Parkinson's disease. J Neurol 2002;249:138-45.

12 Bronnick K, Larsen JP, Aarsland D. Neuropsychiatric disturbances in Parkinson's disease cluster in 5 groups with different prevalence of dementia. Acta Psychiatr Scand 2005;1 12:201-7.

13 Everitt BSLM, Landau S. Cluster analysis. 4th edn. London: Arnold, 2001

14 Emre M, Aarsland D, Albanese A, et al. Rivastigmine for dementia associated with Parkinson's disease. N Engl J Med 2004;351:2509-18.

15 Gibb WR, Lees AJ. The relevance of the Lewy body to the pathogenesis of idiopathic Parkinson's disease. J Neurol Neurosurg Psychiatry 1988:51:745-52.

16 American Psychiatric Association. Diagnostic and statistical manual of mental disorders, 4th edn. Washington, DC: American Psychiatric Association, 1994.

17 Folstein MS, Folstein SE, McHugh PR. "Mini-mental state". A practical method for grading the mental state of patients for the clinician. J Psychiatr Res 1975; 12:189-98.

18 Cummings JL, Mega M, Gray K, et al. The Neuropsychiatric Inventory: comprehensive assessment of psychopathology in dementia. Neurology 1994:44:2308-14.

19 Fahn SRLE. Committee. MotUD. Unified Parkinson's Disease Rating Scale. In: Fahn S, Marsden CD, Calne DMAL, eds. Recent development in Parkinson's disease. Florham Park, NJ, MacMillan Health Care Information, 1987:153-63.

20 Hoehn M, Yahr M. Parkinsonism: onset, progression, and mortality. Neurology 1967;17:427-42.

21 Lyketsos CG, Steinberg M, Tschanz JT, et al. Mental and behavioral disturbances in dementia: findings from the Cache County Study on Memory in Aging. Am J Psychiatry 2000;157:708-14.

22 Tariot PN, Solomon PR, Morris JC, et al. A 5-month, randomized, placebocontrolled trial of galantamine in AD. The Galantamine USA-10 Study Group. Neurology 2000;54:2269-76.

23 Aarsland D, Cummings JL, Larsen JP. Neuropsychiatric differences between Parkinson's disease with dementia and Alzheimer's disease. Int J Geriatr Psychiatry 2001;16:184-91.

24 Hope T, Keene J, Fairburn C, et al. Behaviour changes in dementia. 2: Are there behavioural syndromes, Int J Geriatr Psychiatry 1997;12:1074-8.

25 Ballard CG, Jacoby R, Del Ser T, et al. Neuropathological substrates of psychiatric symptoms in prospectively studied patients with autopsy-confirmed dementia with Lewy bodies. Am J Psychiatry 2004;161:843-9.

26 Ballard C, Piggott $M$, Johnson $M$, et al. Delusions associated with elevated muscarinic binding in dementia with Lewy bodies. Ann Neurol 2000;48:868-76.

27 Aarsland D, Ballard CG, Halliday G. Are Parkinson's disease with dementia and dementia with Lewy bodies the same entity? J Geriatr Psychiatry Neurol 2004; 17:137-45.

28 Schiess MC, Zheng H, Soukup VM, et al. Parkinson's disease subtypes: clinical classification and ventricular cerebrospinal fluid analysis. Parkinsonism Relat Disord 2000;6:69-76.

29 Foltynie T, Brayne CE, Robbins TW, et al. The cognitive ability of an incident cohort of Parkinson's patients in the UK. The CamPalGN study. Brain 2004; 127(P+ 3):550-60. 
30 Janvin C, Aarsland D, Larsen JP, et al. Neuropsychological profile of patients with Parkinson's disease without dementia. Dement Geriatr Cogn Disord 2003; 15:126-31.

31 Weintraub D, Moberg PJ, Culbertson WC, et al. Evidence for impaired encoding and retrieval memory profiles in Parkinson disease. Cogn Behav Neurol 2004; 17:195-200

32 Cummings J. The neuropsychiatry of Alzheimer's disease and related dementias. London, UK: Martin Dunitz, 2003

33 Harding AJ, Broe GA, Halliday GM. Visual hallucinations in Lewy body disease relate to Lewy bodies in the temporal lobe. Brain 2002:125(Pt 2):391-403.

34 Burn DJ. Beyond the iron mask: towards better recognition and treatment of depression associated with Parkinson's disease. Mov Disord 2002; 17:445-54.
35 Tekin S, Mega MS, Masterman DM, et al. Orbitofrontal and anterior cingulate cortex neurofibrillary tangle burden is associated with agitation in Alzheimer disease. Ann Neurol 2001;49:355-61.

36 Burn DJ, Troster Al. Neuropsychiatric complications of medical and surgical therapies for Parkinson's disease. J Geriatr Psychiatry Neurol 2004;17:172-80.

37 Wint DP, Okun MS, Fernandez HH. Psychosis in Parkinson's disease. J Geriatr Psychiatry Neurol 2004;17:127-36.

38 Lyketsos CG, Lopez O, Jones B, et al. Prevalence of neuropsychiatric symptoms in dementia and mild cognitive impairment: results from the cardiovascular health study. JAMA 2002;288:1475-83.

39 Tandberg E, Larsen JP, Aarsland D, et al. The occurrence of depression in Parkinson's disease. A community-based study. Arch Neurol 1996;53:175-9.

40 Hantz P, Caradoc-Davies G, Caradoc-Davies T, et al. Depression in Parkinson's disease. Am J Psychiatry 1994;151:1010-14.

\section{$\mathrm{ECHO}$}

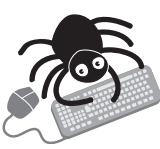

Please visit the Journal of Neurology, Neurosurgery, and Psychiatry website [www.jnnp. com] for a link to the full text of this article.

\section{Misdiagnosis of epilepsy in children}

A survey of children admitted to a tertiary epilepsy centre with difficult to treat paroxysmal events found that $39 \%$ of the children did not have epilepsy. In the retrospective study, case notes of 233 children admitted to the Dianalund Epilepsy Centre-the only tertiary centre of its kind in Denmark-were examined. Their median age was 8 years 6 months (range 8 months to 17 years 8 months) and 54\% were boys.

The referrals were made from local hospitals' paediatric departments in 51\%, other departments in $27 \%$, and from general or specialist practitioners in $22 \%$. Doubt regarding the diagnosis of epilepsy was expressed in the referral note in 17\%. On admission, 86\% of the children were on antiepileptic drug treatment. During admission all children were subjected to a comprehensive intensive observation and 62\% had EEG monitoring.

In total, 87 children (39\%) were found not to have epilepsy. In $30 \%$ of children referred without any doubts about their epilepsy, the diagnosis was found to be wrong. Of the 159 children admitted for the first time, 75 (47\%) were discharged with a diagnosis of non-epileptic seizures. Of 125 admitted for the first time with no doubts about the diagnosis of epilepsy, 44 $(35 \%)$ did not have epilepsy. Staring episodes were the most frequently encountered nonepileptic paroxysmal event. Psychogenic non-epileptic seizures were found in 12 children.

The study shows that the treating physician should be cautious in diagnosis, especially of staring episodes. A diagnostic re-evaluation should be undertaken in difficult cases with continuing paroxysmal events in order to avoid unnecessary drug treatment and restrictions on the child's lifestyle.

$\Delta$ Uldall P, et al. Archives of Diseases in Children 2006;91:219-21 